\title{
Estimation of the Power of the Anomalous Microwave Emission
}

\author{
Kristopher T. Pickens, Giovanna Scarel ${ }^{*}$ (I) \\ Department of Physics and Astronomy, James Madison University, Harrisonburg, VA, USA \\ Email: *scarelgx@jmu.edu
}

How to cite this paper: Pickens, K.T. and Scarel, G. (2020) Estimation of the Power of the Anomalous Microwave Emission. World Journal of Condensed Matter Physics, 10, 105-117.

https://doi.org/10.4236/wjcmp.2020.103007

Received: May 29, 2020

Accepted: June 20, 2020

Published: June 23, 2020

Copyright $\odot 2020$ by author(s) and Scientific Research Publishing Inc. This work is licensed under the Creative Commons Attribution International License (CC BY 4.0).

http://creativecommons.org/licenses/by/4.0/

\section{Open Access}

\begin{abstract}
Context and Background: The product of the electromagnetic (EM) wave's power $P$ times its period $\tau$, i.e. $P \tau$, is the amount of energy conserved in EM wave's absorption in matter. Whether $P \tau$ is the amount of energy conserved in the emission of EM waves from matter is not assessed. Motivation: In this research, we perform a computational study to explore the ability of $P \tau$ to represent the amount of energy conserved in EM wave's emission from matter. Hypothesis: Since the magnitude of the power $P$ of emitted EM waves computed through Larmor's formula for a rotating dipole is excessively small, we alternatively hypothesize that $P \tau$ and the law of conservation of energy can lead to a realistic estimation of $P$. Methods: We estimate the power $P_{\mathrm{AME}}$ of the anomalous microwave emission (AME), a well-characterized radiation generated in the interstellar medium (ISM) by spinning dust grains, and one possible source of contamination of the cosmic microwave background (CMB). For our estimation of $P_{\mathrm{AME}}$, we assume the AME to be generated in a molecular cloud mostly populated by spinning silicate nanoparticles (SSNs) or polycyclic aromatic hydrocarbon (PAH) spinning dust grains. Indeed, SSNs and PAHs are listed among the most probable sources of AME, and their characteristics are well-known. We discriminate between realistic and non-realistic values of $P_{\mathrm{AME}}$ based upon the magnitude of two parameters that depend on $P_{\mathrm{AME}}$ : the significant distance $z$, and the time of photon production $T$. The parameter $\mathrm{z}$ is the space interval from the spinning dust grain within which the spinning dust grain's electric field is effective. Results: Using the information available for AME, SSNs and PAHs, we estimate the power $P_{\mathrm{AME}}$ using both Larmor's formula and $P \tau$. We compare and comment the results obtained for $z$ and $T$. Conclusions: Our study highlights the effectiveness of $P \tau$ over Larmor's formula in providing a realistic value of $P_{\mathrm{AME}}$. This finding might have consequences in quantum technology of single photon detection and production.
\end{abstract}




\section{Keywords}

Anomalous Microwave Emission, Spinning Dust, Power of Emitted Radiation

\section{Introduction}

Is $P \tau$, i.e. the product of light's power $P$ times its period $\tau$, the amount of energy conserved in the emission of electromagnetic (EM) waves from matter? This outstanding question is not addressed in the current scientific literature. In this research, we computationally tackle this problem. Before outlining our strategy, we summarize the status of this field of research. Recently, Boone et al. [1] found that $P \tau$ is the amount of energy conserved in the absorption of EM waves in matter. To date, this finding is quantitatively proven in the absorption of visible and infrared (IR) light by capacitors [1] [2], by thin films in IR spectra [3], by the retina in vertebrate's vision [4], by molecules in photoredox catalysis reactions [5], in photo-thermoelectric devices [5], and by neurons under two-photon excitation [5]. On the contrary, only some indirect evidence that $P \tau$ could work in emission of EM waves exists, and is offered by the radiation from free electrons [6] [7]. From the computational and the experimental points of view, one of the difficulties in studying EM wave's emission lies in finding sources of EM waves with a well-determined emitted power and frequency $v$ (or period $\tau=v^{-1}$ ). In response to this difficulty, in our computational study we investigate the anomalous microwave emission (AME) radiated by spinning silicate nanoparticles (SSNs) or polycyclic aromatic hydrocarbon (PAH) spinning dust grains in the interstellar medium (ISM). With the available information, we determine the emitted power $P_{\mathrm{AME}}$ and the period $\tau_{\mathrm{AME}}$. On one hand, estimating $\tau_{\mathrm{AME}}$ is straightforward, since $\tau_{\mathrm{AME}}=1 / v_{\mathrm{AME}}$, and $v_{\mathrm{AME}}$ is well-known to peak in intensity in the frequency interval between 10 and $100 \mathrm{GHz}$ [8] [9]. On the other hand, there are two routes to estimating $P_{\mathrm{AME}}$ : one is through the classical electrodynamics Larmor's formula; another is through $P \tau$ and the law of conservation of energy. We determine $P_{\mathrm{AME}}$ from both routes, and discriminate whether the obtained values of $P_{\mathrm{AME}}$ are realistic or not through the values of two $P_{\mathrm{AME}}$-dependent parameters. These two parameters are: $Z$, the significant distance from a spinning dust grain within which its electric field is effective, and $T$, the time of photon production. Discussing the values of $z$ and $T$ will enable us to draw conclusions on the ability of $P_{\mathrm{AME}} \tau_{\mathrm{AME}}$ to represent the amount of energy conserved in the emission of AME from spinning dust grains.

Before diving into the details of our investigation, let us outline an overview on the AME. Generally, the production of AME is ascribed to the dipole moment of the spinning dust grains in the ISM [10] [11]. The value of the dipole moment, the shape, size, composition, and the density of the AME are well-known. Indeed, the AME is one of the sources of contamination of the cosmic microwave background $(\mathrm{CMB})$. Thus, the AME needs to be subtracted from the CMB 
signal in order to enable the extraction of information of cosmological relevance from the CMB [12]. For this reason, in the past twenty years the AME received large attention from many research groups.

Since long time, the PAH spinning dust grains are considered the main contributors to the generation of the AME [9] [11]. More recently, however, the lack of correlation between AME and PAH spinning dust grains [13] prompted the research community to consider also other sources. Among them, SSNs [14], magnetic grains [15], and diamond nanoparticles [16] were proposed. In this research, we focus first on SSNs as spinning dust grains generating the AME, and utilize the information on SSN's size, shape, composition, density, and electric dipole available from Hoang and Lazarian [15]. The amount of SSNs in the ISM is unknown and might be even very small, however the abundant information on SSNs will easily enable us sketching a rough prediction on $P_{\mathrm{AME}}$ and the parameters $z$ and $T$. We then compute $P_{\mathrm{AME}}$ and the parameters $z$ and $T$ also for $\mathrm{PAH}$ spinning dust grains and compare the results with those obtained for the SSNs.

The AME was first discovered in the late 1990s [17] [18] [19], and further characterized, through direct observations made possible by the Planck satellite. The AME was clearly identified in the Perseus and $\rho$ Ophiuchi molecular clouds, and in two compact radiation-emitting dust regions known as AME-G173.6 + 2.8 and AME-G107.1 + 5.2 [8]. Additional sources were described in a later publication [9]. Hoang et al. [20] investigated other sources of AME, e.g. those in Herbig A- and B-type stars, and in T-Tauri young variable stars, which are located near molecular clouds. The existence of a relationship between AME and spinning dust grains in the ISM was first proposed by Draine \& Lazarian [10] [11]. More information on AME can be found in numerous publications, e.g. [21]-[30].

As mentioned above, there is general consensus among the authors regarding the frequency range of $v_{\mathrm{AME}}$, which is in the 10 to $100 \mathrm{GHz}$ interval. Some authors further highlight that the magnitude of $v_{\mathrm{AME}}$ is of the same order of the rotational frequency $\omega_{\text {rot }}$ of the spinning dust grains that generate the AME [31] [32] [33]. Specifically:

$$
v_{\mathrm{AME}} \approx\left(\frac{5}{3}\right)^{\frac{1}{4}} \omega_{\mathrm{rot}} .
$$

Equation (1) signifies that the rotational frequency $\omega_{\text {rot }}$ is in the $\mathrm{GHz}$ range. It is very likely that such high rotational frequency is enabled by the small size of the spinning dust grains, whose radius is typically of the order of $\mathrm{nm}$, or a fraction of it [11] [14].

Almost all authors report that Larmor's formula offers a good estimation of $P_{\mathrm{AME}}$, the power of the AME emitted by spinning dust grains. Various authors [10] [11] [14] [16] [20] [33] [34] report Larmor's formula in Gaussian units as:

$$
P=\frac{2}{3} \frac{\mu^{2} \omega_{\mathrm{rot}}^{4}}{c^{3}},
$$


where $\mu$ is the electric dipole moment in units of $\mathrm{D}$ (Debye, corresponding to $3.33564 \times 10^{-30} \mathrm{C} \cdot \mathrm{m}$ ) of the spinning dust grains, and $c$ is the speed of light in vacuum approximated to $3 \times 10^{8} \mathrm{~m} \cdot \mathrm{s}^{-1}$. In the available literature, however, actual values of $P_{\mathrm{AME}}$ are extremely difficult to find, and the few reported values are very small: e.g. $P_{\mathrm{AME}} \sim 10^{-47} \mathrm{~W}$ per grain [15]. This lack of information on $P_{\mathrm{AME}}$ prompts us to compute the value of $P_{\mathrm{AME}}$ from Larmor's formula expressed with Standard International (S.I.) units. The small values we obtained stimulate us to follow an alternative route to compute $P_{\mathrm{AME}}$ by applying $P \tau$ and the law of conservation of energy.

To apply $P \tau$ and the law of conservation of energy to AME's emission, we first identify the types of energy of the spinning dust grains that are transformed into the EM wave's energy. We hypothesize that the spinning dust grain's electrical and mechanical energies fulfill such task. The electrical energy is due to the electric dipole moment $\mu$ of the spinning dust grain, while the mechanical energy involves its moment of inertia $I$. In order to significantly and effectively contribute to the conserved energy $P \tau$, the spinning dust grain's electrical and mechanical energies are required to be of the same order of magnitude of $P \tau$. To compute the electrical and mechanical energies, we utilize the known information on the spinning dust grain's size, shape, composition, density, and electric dipole.

\section{Methods}

\subsection{P $\tau$ and the Law of Conservation of Energy in the Interaction between EM Waves and Matter}

It is well established that the power $P$ radiated by a rotating dipole is given by Larmor's formula, which we will discuss in the next section. On the other hand, it is less straightforward to establish how much is the energy conserved when energy is transferred to a radiated field or EM wave by an accelerating charge, or a spinning dipole. Is this energy equal to the energy $h v$ of a photon, where $h$ is Planck's constant $\left(6.63 \times 10^{-34} \mathrm{~J} \cdot \mathrm{s}\right)$ ? Or, is this energy equal to that of $\mathrm{n}$ photons? If so, what is the relationship between the emitted power $P$ and the number $n$ of photons of the emitted EM wave at constant frequency $v$ ? These questions prompted us to investigate whether it is possible to use the law of conservation of energy to respond. To this end, we assume that an accelerating charge, a spinning dipole or, in general, matter capable of emitting EM waves, have electrical energy $\left(E_{\mathrm{el}}\right)$, thermal energy $\left(E_{\mathrm{th}}\right)$, and mechanical energy $\left(E_{\mathrm{mec}}\right)$. These energies contribute to the generation of the radiated field or EM wave. Vice-versa, the energy $E_{\mathrm{EM}}$ of the radiated field depends on them. Thus, for the law of conservation of energy, $E_{\mathrm{el}}+E_{\mathrm{th}}+E_{\mathrm{mec}}=E_{\mathrm{EM}}$, assuming that the energy dissipated during the energy transfer process between the radiating object and the EM wave is negligible. We can easily give an analytical expression to $E_{\mathrm{e}}, E_{\mathrm{th}}$, and $E_{\mathrm{mec}}$ and estimate their magnitude. However, it is less straightforward to establish an analytical expression and estimate for the magnitude of $E_{\mathrm{EM}}$. Imagining that the radiating 
object emits at a constant frequency $v$, we have various options for $E_{\mathrm{EM}}: h v, n h v$, or $P \Delta t$. Here $\Delta t$ is an arbitrary time interval. However, using the law of conservation of energy in absorption phenomena, where the power of the EM waves absorbed by a system is known, it was found that $\Delta t=\tau=v^{-1}$, where $\tau$ is the period of the EM wave and $v$ its frequency. The equation $E_{\mathrm{EM}}=P \tau$ captures the order of magnitude of the variables affected by the absorption process, and justifies the power-dependent response of the absorption [1] [2] [3] [4] [5]. Here, we hypothesize that the approach used in absorption phenomena is true also in emission phenomena. Thus, we assume $E_{\mathrm{EM}}=P \tau$, and test the validity of this choice. We appreciate the fact that $P \tau$ changes continuously because, in agreement with Draine and Lazarian [10] [11] we want to describe the phenomenon of EM wave emission by charges and dipoles in the realm of classical physics.

\subsection{Larmor's Formula}

Larmor's formula in Equation (2) is expressed in Gaussian units. In order to compute $P_{\mathrm{AME}}$ in S.I. units, i.e. in Watts (W), we transform Larmor's formula as follows:

$$
P_{\mathrm{AME}}=\frac{2}{3} \frac{k \mu^{2} \omega_{\mathrm{rot}}^{4}}{c^{3}} .
$$

In Equation (3), the dipole moment $\mu$ in units of $\mathrm{D}$ (Debye) is multiplied by $k^{1 / 2}$, where $k$ is Coulomb's constant $\left(8.9910^{9} \mathrm{~N} \cdot \mathrm{m}^{2} \cdot \mathrm{C}^{-2}\right)$.

\subsection{Characteristic Properties of the SSNs and PAH Spinning Dust Grains}

In our calculations, for simplicity, we assume that the AME is generated by spherical SSNs with $\mathrm{MgSiO}_{3}$ composition [14]. We consider the number of atoms $N$ in one SSN to be $N=7 N_{\mathrm{Si}}=417.74 a_{-7}^{3}$, where $a_{-7}=a / 10^{-7} \mathrm{~cm}$, and $a=0.4 \mathrm{~nm}$ is the radius of a spherical SSN [14]. With this choice of a, it turns out that $N=27$. The dipole moment for such an SSN is $\mu=12.2 \mathrm{D}$, where $D=3.33564 \times 10^{-30} \mathrm{C}$ $\mathrm{m}$, and the SSN's density is $\delta \approx 4 \mathrm{~g} \mathrm{~cm}^{-3}$ [14]. We estimate the mass of each spherical SSN to be $m=\delta \frac{4}{3} \pi a^{3}=1.07 \times 10^{-24} \mathrm{~kg}$, and its moment of inertia $I$ to be $I=\frac{2}{5} m a^{2}=6.85 \times 10^{-44} \mathrm{~kg} \cdot \mathrm{m}^{2}$. The characteristic properties of the SSNs are summarized in Table 1. Those of cylindrical PAH spinning dust grains, taken from Draine and Lazarian [11], are collected in Table 2.

Table 1. Summary of the characteristics of a spinning silicate nanoparticle (SSN) taken from Ref. [14].

\begin{tabular}{cccccc}
\hline$N$ & $\mu$ & $\delta$ & $m$ & $I$ & $\omega_{\text {rot }}$ \\
\hline 27 & $12.2 \mathrm{D}$ & $4 \mathrm{~g} \cdot \mathrm{cm}^{-3}$ & $1.07 \times 10^{-24} \mathrm{~kg}$ & $6.85 \times 10^{-44} \mathrm{~kg} \cdot \mathrm{m}^{2}$ & $30 \mathrm{GHz}$ \\
\hline
\end{tabular}

$N$ is the average number of atoms in one SSN, $\mu$ is the SSN's dipole moment in units of D (Debye), $\delta$ its density, $m$ its mass, $I$ its moment of inertia, and $\omega_{\text {rot }}$ its rotational frequency. For our calculations, we assume spherical SSNs with radius $\mathrm{a}=0.4 \mathrm{~nm}$. 
Table 2. Summary of the characteristics of polycyclic aromatic hydrocarbon (PAH) spinning dust grains taken from Draine \& Lazarian [11].

\begin{tabular}{cccccc}
\hline$N$ & $\mu$ & $\delta$ & $m$ & $I$ & $\omega_{\text {rot }}$ \\
\hline 25.43 & $2 \mathrm{D}$ & $2 \mathrm{~g} \cdot \mathrm{cm}^{-3}$ & $0.59 \times 10^{-24} \mathrm{~kg}$ & $3.80 \times 10^{-44} \mathrm{k} \cdot \mathrm{gm}^{2}$ & $30 \mathrm{GHz}$ \\
\hline
\end{tabular}

$N$ is the average number of atoms in one PAH spinning dust grain, $\mu$ is a PAH's average dipole moment in units of D (Debye), $\delta$ its density, $m$ its mass, $I$ its moment of inertia, and $\omega_{\text {rot }}$ its rotational frequency. For our calculations, we assume cylindrical PAH spinning dust grain with radius $a=0.36 \mathrm{~nm}$ and height $h=2 a$.

\section{Results}

\subsection{Power of the Emitted AME Estimated from Larmor's Formula}

We assume for simplicity that, for the SSNs, $\omega_{\text {rot }}=v_{\mathrm{AME}}=30 \mathrm{GHz}$. By multiplying the dipole moment $\mu$ by the number $N$ of atoms in the SSNs, we obtain $\mu_{\text {effective }}=N \mu$. By replacing $\mu$ with $\mu_{\text {effective }}=N \mu$ in Equation (3), we estimate $P_{\mathrm{AME}}$ $=0.22 \times 10^{-27} \mathrm{~W}$. Reasoning in the same way, for PAH spinning dust grains we obtain $P_{\mathrm{AME}}=5.24 \times 10^{-30} \mathrm{~W}$.

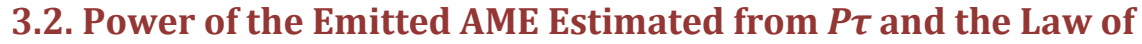 Conservation of Energy}

An SSN is charged and gives rise to an electric dipole [10] [11], which rotates at a rate of $\omega_{\text {rot }} \approx 30 \mathrm{GHz}$, as assumed in Section 3.1. We hypothesize that the energy $P_{\mathrm{AME}} \tau_{\mathrm{AME}}$ of the AME, where $\tau_{\mathrm{AME}}=\frac{1}{v_{\mathrm{AME}}}$, is generated by the spinning of the SSNs with rotational kinetic energy $E_{\text {rot }}=\frac{1}{2} I \omega_{\text {rot }}^{2}$ plus an electric energy contribution $E_{\text {dipole }}$, due to the dipole $\mu_{\text {effective }}=N \mu$ of the SSN, defined in Section 3.1, such that:

$$
E_{\text {dipole }}=\frac{1}{4 \pi \varepsilon_{0}} N^{2} \mu^{2} \frac{1}{z^{3}} .
$$

Thus $E_{\text {rot }}+E_{\text {dipole }}=P_{\mathrm{AME}} \tau_{\mathrm{AME}}$, in agreement with our discussion in Section 2.1, or:

$$
\frac{1}{2} I \omega_{\mathrm{rot}}^{2}+\frac{1}{4 \pi \varepsilon_{0}} N^{2} \mu^{2} \frac{1}{z^{3}}=P_{\mathrm{AME}} \tau_{\mathrm{AME}} .
$$

In Equation (4) and Equation (5), $z$ is the significant distance from the SSN within which the SSN's electric field is effective. The constant $\varepsilon_{0}$ is the dielectric permittivity in vacuum $\left(8.854 \times 10^{-12} \mathrm{Fm}^{-1}\right)$. In Equation (5), $P_{\mathrm{AME}} \tau_{\mathrm{AME}}$ is the amount of energy conserved in the emission of EM waves from the SSN, in agreement with our discussion in Section 2.1. To be significant, both $E_{\text {rot }}$ and $E_{\text {dipole }}$ are required to be of the same order of magnitude of $P_{\mathrm{AME}} \tau_{\mathrm{AME}}$. This constraint enables us estimating the magnitude of both $P_{\mathrm{AME}}$ and $z$. We estimate the power $P_{\mathrm{AME}}$ as $P_{\mathrm{AME}} \approx E_{\mathrm{rot}} / \tau_{\mathrm{AME}}$. Since $\tau_{\mathrm{AME}}=\frac{1}{v_{\mathrm{AME}}}$, and $\omega_{\mathrm{rot}}=v_{\mathrm{AME}}=30 \mathrm{GHz}$, it turns out that $P_{\mathrm{AME}}=0.93 \mathrm{pW}$. Reasoning in the same way, for PAH spinning dust grains we obtain $P_{\mathrm{AME}}=0.51 \mathrm{pW}$. We estimate $z$ in Section 3.3. 


\subsection{Significant Distance $\mathrm{z}$ from Power $\boldsymbol{P}_{\mathrm{AME}}$ Derived through Larmor's Formula and $P \tau$}

Using Larmor's formula, the power $P_{\mathrm{AME}}$ emitted by the SSNs is $P_{\mathrm{AME}}=0.22 \times 10^{-27}$ $\mathrm{W}$, as discussed in Section 3.1. In this case, $P_{\mathrm{AME}} \tau_{\mathrm{AME}}=0.0068 \times 10^{-36} \mathrm{~J}$ and, using Equation (4) expressed as $z^{3}=\frac{1}{4 \pi \varepsilon_{0}} N^{2} \mu^{2}\left(E_{\text {dipole }}\right)^{-1}$ and $P_{\mathrm{AME}} \tau_{\mathrm{AME}} \approx E_{\text {dipole }}$, we obtain $z=1.17 \mathrm{~cm}$. Reasoning in the same way, for PAH spinning dust grains we obtain $Z=1.14 \mathrm{~cm}$. On the other hand, using $P \tau$ and the law of conservation of energy, for SSNs we estimate $P_{\mathrm{AME}}=0.93 \mathrm{pW}$. Then,

$E_{\text {dipole }} \approx P_{\mathrm{AME}} \tau_{\mathrm{AME}}=0.031 \times 10^{-21} \mathrm{~J}$, and $z=70.5 \mathrm{~nm}$. Reasoning in the same way, for PAH spinning dust grains we obtain $Z=24.88 \mathrm{~nm}$.

\subsection{Time of Photon Production T Obtained with Larmor's Formula and $P \tau$}

For SSNs, with $P_{\mathrm{AME}}=0.22 \times 10^{-27} \mathrm{~W}$ from Larmor's formula, and $0.93 \mathrm{pW}$ from $P \tau$ and the law of conservation of energy, we estimate the time of production $T$ of photons with energy $h v_{\mathrm{AME}}=19.89 \times 10^{-24} \mathrm{~J}$ as $T=h v_{\mathrm{AME}} / P_{\mathrm{AME}}$. Here, $h$ is Planck's constant $\left(6.63 \times 10^{-34} \mathrm{~J} \cdot \mathrm{s}\right)$. It turns out that $T=1.05$ days with $P_{\mathrm{AME}}$ from Larmor's formula, and $T=21.4$ ps with $P_{\mathrm{AME}}$ from $P \tau$. Analogously, for PAH spinning dust grains, we obtain $T=1.44$ months with $P_{\text {AME }}$ from Larmor's formula, and $T=39.0$ ps with $P_{\text {AME }}$ from $P \tau$.

\section{Discussion}

So far, we estimated the power $P_{\mathrm{AME}}$ and the parameters $Z$ and $T$ of the AME produced by SSNs and PAH spinning dust grains. Our results are summarized in Table 3 and Table 4. We now discuss the consequences of the different values of $P_{\text {AME }}, Z$ and $T$ obtained from Larmor's formula and from $P \tau$ and the law of conservation of energy.

\subsection{The Power $P_{\mathrm{AmE}}$}

As reported in Section 3.1 and 3.2, and in Table 3, for SSNs we estimate $P_{\mathrm{AME}}=$ $0.22 \times 10^{-27} \mathrm{~W}$ from Larmor's formula, and $P_{\mathrm{AME}}=0.93 \mathrm{pW}$ from $P \tau$ and the law of conservation of energy. These two values are 15 orders of magnitude apart. For the PAH spinning dust grains the difference is 18 orders of magnitude. This difference requires some comment. Larmor's formula in Equation (3) is a widely known and well-established result of classical electrodynamics. It depends on two constants: Coulomb's constant $k=8.99 \times 10^{9} \mathrm{~N} \cdot \mathrm{m}^{2} \cdot \mathrm{C}^{-2}$, and the speed of light in vacuum, $c=3 \times 10^{8} \mathrm{~m} \cdot \mathrm{s}^{-1}$. The rotational frequency $\omega_{\text {rot }}$ and the dipole moment $\mu$ are the two variables in Larmor's formula. Both these variables are properties of the spinning dust grain, which are fixed. Indeed, the rotational frequency $\omega_{\text {rot }}$ is required to be of a magnitude comparable to that of the frequency of the emitted AME. Moreover, the dipole moment $\mu$ varies in a small range of values (see e.g. [11]), and its unit in the S.I. system is defined by the conversion 
Table 3. Summary of the characteristics of the AME produced by spherical spinning silicate nanoparticles (SSNs).

\begin{tabular}{cccc}
\hline & $P_{\text {AME }}(\mathrm{W})$ & $Z(\mathrm{~m})$ & $T$ \\
\hline Larmor & $0.22 \times 10^{-27}$ & $1.17 \times 10^{-2}$ & 1.05 days \\
$P \tau$ & $0.93 \times 10^{-12}$ & $70.5 \times 10^{-9}$ & $21.4 \mathrm{ps}$ \\
\hline
\end{tabular}

We list the power $P_{\mathrm{AME}}$ of the anomalous microwave emission (AME) emitted by the SSNs. We estimate $P_{\mathrm{AME}}$ with Larmor's formula and using $P \tau$ and the law of conservation of energy. We also report the parameters $z$ and $T$. The parameter $z$ is the significant distance from the SSN's dipole within which the SSN's electric field is effective. The parameter $T$ is the time of production of photons with energy $h v_{\mathrm{AmE}}=19.89 \times$ $10^{-24} \mathrm{~J}$.

Table 4. Summary of the characteristics of the AME produced by cylindrical polycyclic aromatic hydrocarbon $(\mathrm{PAH})$ spinning dust grains.

\begin{tabular}{cccc}
\hline & $P_{\text {AME }}(\mathrm{W})$ & $Z(\mathrm{~m})$ & $T$ \\
\hline Larmor & $5.24 \times 10^{-30}$ & $1.14 \times 10^{-2}$ & 1.44 months \\
$P \tau$ & $0.51 \times 10^{-12}$ & $24.88 \times 10^{-9}$ & 39.0 ps \\
\hline
\end{tabular}

We list the power $P_{\mathrm{AME}}$ of the anomalous microwave emission (AME) emitted by the PAH spinning dust grains. We estimate $P_{\mathrm{AME}}$ with Larmor's formula and using $P \tau$ and the law of conservation of energy. We also report the parameters $z$ and $T$. The parameter $z$ is the significant distance from the PAH dipole within which the PAH's electric field is effective. The parameter $T$ is the time of production of photons with energy $h v_{\mathrm{AME}}=19.89 \times 10^{-24} \mathrm{~J}$.

factor $D=3.33564 \times 10^{-30} \mathrm{C} \cdot \mathrm{m}$. These constraints on $\omega_{\mathrm{rot}}$ and $\mu$ impose a low degree of freedom to the value of $P_{\mathrm{AME}}$. On the other hand, Equation (5) for $P \tau$ and the law of conservation of energy depends on the properties of the spinning dust grain: the rotational frequency $\omega_{\text {rot }}$ the dipole moment $\mu$, but also the mass and the geometry (size and shape) of the spinning dust grain. Thus, the power $P_{\mathrm{AME}}$ can be estimated with a larger degree of freedom (mass, size, shape) with $P \tau$ than with Larmor's formula.

\subsection{The Significant Distance $z$}

We recall that $z$ is the significant distance from the spinning dust grain within which the spinning dust grain's electric field is effective. The significant distance $z$ of $z=1.17 \mathrm{~cm}$ for SSNs obtained, e.g., from $P_{\mathrm{AME}}$ calculated through Larmor's formula implies that the electric field around the SSN is effective within a large radius. In this case, in dense molecular clouds populated by SSNs, the electric field of one SSN may couple with the electric fields of a large number of neighboring SSNs, slowing down their frequency of rotation $\omega_{\text {rot. }}$. This hypothesis is realistic because the typical number density of molecules in a molecular cloud is about $3 \times 10^{8} \mathrm{~m}^{-3}$ [35]. Therefore, approximately 2000 molecules may dwell in a sphere of radius $1.17 \mathrm{~cm}$, and thus also hundreds of SSNs (assuming that molecular clouds are predominantly populated by SSNs). In these conditions, the interaction among the electric fields of the SSNs could lead to decreasing their frequency of rotation $\omega_{\mathrm{rot}}$, thus interrupting the production of AME at $30 \mathrm{GHz}$. On the other hand, a value of $z=70.5 \mathrm{~nm}$, obtained from $P_{\text {AME }}$ estimated 
through $P \tau$ and the law of conservation of energy, implies that the electric field around the SSN is effective within a short radius. In this case, the coupling of the electric fields of neighboring SSNs even in a dense molecular cloud is negligible, thus minimizing the probability of reducing the frequency of rotation $\omega_{\text {rot }}$ of the surrounding SSNs and interrupting AME's production. Indeed, the typical number density of molecules in a molecular cloud of about $3 \times 10^{8} \mathrm{~m}^{-3}$ [35] implies that only $0.440 \times 10^{-12}$ molecules (notice the negative exponent!) may dwell in a sphere of radius $70.5 \mathrm{~nm}$, minimizing the probability to find SSNs at a close distance, of the order of $\mathrm{nm}$, one from the other. This statement follows the assumption that molecular clouds are predominantly populated by SSNs. Reasoning in the same way, we reach similar conclusions for PAH spinning dust grains.

\subsection{The Time of Photon Production $T$}

For SSNs, with $P_{\mathrm{AME}}=0.22 \times 10^{-27} \mathrm{~W}$ calculated through Larmor's formula, we estimate a time of photon production of $T=1.05$ days. Considering that the period of AME photons is $\tau_{\mathrm{AME}}=33 \mathrm{ps}, T=1.05$ days corresponds to $2.7 \times 10^{15} \mathrm{pe}-$ riods, if the photon would be able to keep a coherent phase over all this time. In addition, given that photons move at the speed of $3 \times 10^{8} \mathrm{~ms}^{-1}$, a time $T=1.05$ days signifies that the "forming" photon travels away from its origin's point by about $27 \times 10^{9} \mathrm{~km}$. What do $2.7 \times 10^{15}$ periods mean for a "forming" photon? What does it mean such a delocalization from the photon's origin point? What happens to the "forming" photon if it interferes with other photons or "forming" photons? What would happen to its coherence? On the other hand, with $P_{\mathrm{AME}}=$ $0.93 \mathrm{pW}$ estimated through $P \tau$ and the law of conservation of energy, we obtain a time of photon production $T=21.4 \mathrm{ps}$, which is of the same order of magnitude of $\tau_{\mathrm{AME}}=33 \mathrm{ps}$. The $\mathrm{T}$ magnitude of $21.4 \mathrm{ps}$ signifies that the "forming" photon travels away from its origin point by about $6.5 \mathrm{~mm}$, in a hypothetical spherical volume in which approximately 350 other "forming" photons might be found. Thus, in the interior of the hypothetical spherical volume, the photons experience a favorable environment where to develop their characteristic frequency. Similar considerations can be applied to PAH spinning dust grains.

\subsection{Comparison with Polycyclic Aromatic Hydrocarbon (PAH) Spinning Dust Grains}

In Table 2 we collect the information on $N, \mu, \delta, m, I, \omega_{\text {rot }}$ and the radius a for cylindrical PAH spinning dust grains, which are listed among the possible sources of AME [9] [11]. For our calculations, we assume cylindrical PAH spinning dust grain with radius $a=0.36 \mathrm{~nm}$ and height $h=2 a$. We then compute $P_{\text {AME }}$ with Larmor's formula and with $P \tau$ and the law of conservation of energy. In addition, we estimate the parameters $z$ and $T$, and report the results in Table 4. The $P_{\mathrm{AME}}$ from Larmor's formula is three orders of magnitude smaller for PAH spinning dust grains than it is for SSNs, while the time of photon production $T$ for PAH spinning dust grains reaches 1.44 months. The significant distance $z$ for PAH spinning dust grains is of the same order of magnitude as that 
for the SSNs. We ascribe the large difference between the values of $P_{\mathrm{AME}}$ and $T$ obtained for PAH spinning dust grains and SSNs to the difference in magnitude of their dipole moment $\mu$. On the other hand, the $P_{\mathrm{AME}}$ from $P \tau$ and the law of conservation of energy is of the same order of magnitude of that of SSNs, so are also $z$ and $T$. These findings suggest that small variations of composition, size and shape of the spinning dust grains do not affect significantly the characteristics of the AME generated by spinning dust grains.

\section{Conclusion}

The purpose of this research is to find the energy conserved in the emission of electromagnetic (EM) waves from matter. We tackle this problem through a computational investigation in which we estimate the power $P_{\mathrm{AME}}$ of the anomalous microwave emission (AME) generated by spinning dust grains in the interstellar medium (ISM). We first compute $P_{\mathrm{AME}}$ through Larmor's formula obtaining $P_{\mathrm{AME}} \leq 10^{-27} \mathrm{~W}$. In this case, the estimated significant distance $z$ of the electric field around the spinning dust grains suggests couplings between the spinning dust grains that might slow down their rotations. Moreover, the obtained time of photon production T suggests that the "forming" photons could suffer severe interferences while forming. We then compute $P_{\mathrm{AME}}$ from $P \tau$, i.e. the product of the EM wave's power $P$ times its period $\tau$. In this case, we estimate $P_{\mathrm{AME}} \sim \mathrm{pW}$. Such $P_{\mathrm{AME}}$ gives rise to a value of $z$ small enough to prevent coupling with the electric fields of neighboring grains. Moreover, with $P_{\mathrm{AME}} \sim \mathrm{pW}$, the magnitude of $T$ is small enough to offer to the "forming" photons a favorable environment where to develop their characteristic frequency. These results are independent of the composition of the spinning dust grains and validate the hypothesis that $P \tau$ is the amount of energy conserved in the interaction between EM waves and matter in emission phenomena. Our results may impact a vast range of light-matter interaction phenomena. We envision consequences, for example, for the quantum technology of single photon detection and production, which needs further investigation.

\section{Acknowledgements}

The authors wish to acknowledge the support from the Department of Physics and Astronomy, James Madison University (JMU).

\section{Conflicts of Interest}

The authors declare no conflicts of interest regarding the publication of this paper.

\section{References}

[1] Boone, D.E., Jackson, C.H., Swecker, A.T., Hergenrather, J.S., Wenger, K.S., Kokhan, O., Terzić, B. Melnikov, I., Ivanov, I.N., Stevens, E.C. and Scarel, G. (2018) Probing the Wave Nature of Light-Matter Interaction. World Journal of Condensed Matter Physics, 8, 62-89. https://doi.org/10.4236/wjcmp.2018.82005 
[2] Gordon, A.L. and Scarel, G. (2018) Interaction in the Steady State between Electromagnetic Waves and Matter. World Journal of Condensed Matter Physics, 8, 171-184. https://doi.org/10.4236/wjcmp.2018.84012

[3] Scarel, G. and Stevens, E.C. (2019) The Effect of Infrared Light's Power on the Infrared Spectra of Thin Films. World Journal of Condensed Matter Physics, 9, 1-21. https://doi.org/10.4236/wjcmp.2019.91001

[4] Scarel, G. (2019) Quantum and Non-Quantum Formulation of Eye's Adaptation to Light's Intensity. World Journal of Condensed Matter Physics, 9, 62-74. https://doi.org/10.4236/wjcmp.2019.93005

[5] Scarel, G. (2019) The Role of $\mathrm{P} \tau$ in the Photothermoelectric Effect and in Photoredox Catalysis Reactions. World Journal of Condensed Matter Physics, 9, 91-101. https://doi.org/10.4236/wjcmp.2019.94007

[6] Yang, Y., Massuda, A., Roques-Carmes, C., Kooi, S.E., Christensen, T., Johnson, S.G., Joannopoulos, J.D., Miller, O.D., Kaminer, I. and Soljačić, M. (2018) Maximal Spontaneous Photon Emission and Energy Loss from Free Electrons. Nature Physics, 14, 894-899. https://doi.org/10.1038/s41567-018-0180-2

[7] Roques-Carmes, C., Kooi, S.E., Yang, Y., Massuda, A., Keathley, P.D., Zaidi, A., Yang, Y.J., Joannopoulos, J.D., Berggren, K.K., Kaminer, I. and Soljačić, M. (2019) Towards Integrated Tunable All-Silicon Free-Electron Light Sources. Nature Communications, 10, 3176. https://doi.org/10.1038/s41467-019-11070-7

[8] Ade, P.A.R., et al. (Planck Collaboration) (2011) Planck Early Results. XX. New Light on Anomalous Microwave Emission from Spinning Dust Grains. Astronomy and Astrophysics, 536, A20-A37.

[9] Ade, P.A.R., et al. (Planck Collaboration) (2014) Planck Intermediate Results. A Study of Anomalous Microwave Emission in Galactic Clouds. Astronomy and Astrophysics, 565, A103-A131.

[10] Draine, B.T. and Lazarian, A. (1998) Diffuse Galactic Emission from Spinning Dust Grains. The Astrophysical Journal, 494, L19-L22. https://doi.org/10.1086/311167

[11] Draine, B.T. and Lazarian, A. (1998) Electric Dipole Radiation from Spinning Dust Grains. The Astrophysical Journal, 508, 157-179. https://doi.org/10.1086/306387

[12] Ichiki, K. (2014) CMB Foreground: A Concise Review. Progress in Theoretical and Experimental Physics, 2014, 06B109. https://doi.org/10.1093/ptep/ptu065

[13] Hensley, B.S., Draine, B.T. and Meisner, A.M. (2016) A Case against Spinning PAHs as the Source of the Anomalous Microwave Emission. The Astrophysical Journal, 827, 45-58. https://doi.org/10.3847/0004-637X/827/1/45

[14] Hoang, T. Vinh, N.-A. and Lan, N.-Q. (2016) Spinning Dust Emission from Ultra-Small Silicates: Emissivity and Polarization Spectrum. The Astrophysical Journal, 824, 18-29. https://doi.org/10.3847/0004-637X/824/1/18

[15] Hoang, T. and Lazarian, A. (2016) Polarization of Magnetic Dipole Emission and Spinning Dust Emission from Magnetic Nanoparticles. The Astrophysical Journal, 821, 91-110. https://doi.org/10.3847/0004-637X/821/2/91

[16] Greaves, J.S., Scaife, A.M.M., Frayer, D.T., Green, D.A., Mason, B.S. and Smith, A.M.S. (2018) Anomalous Microwave Emission from Spinning Nanodiamonds around Stars. Nature Astronomy, 2, 662-667. https://doi.org/10.1038/s41550-018-0495-Z

[17] Kogut, A., Banday, A.J., Bennett, C.L., Górski, K.M., Hinshaw, G., Smoot, G.F. and Wright, E.L. (1996) Microwave Emission at High Galactic Latitudes in the Four-Year DMR Sky Map. The Astrophysical Journal, 464, L5-L9. https://doi.org/10.1086/310072 
[18] de Oliveira-Costa, A., Kogut, A., Devlin, M.J., Netterfield, C.B., Page, L.A. and Wollack, E.J. (1997) Galactic Microwave Emission at Degree Angular Scales. The Astrophysical Journal, 527, L17-L20. https://doi.org/10.1086/310684

[19] Leitch, E.M., Readhead, A.C.S., Pearson, A.C.S. and Myers, S.T. (1997) An Anomalous Component of Galactic Emission. The Astrophysical Journal, 486, L23-L26. https://doi.org/10.1086/310823

[20] Hoang, T., Lan, N.Q., Vinh, N.-A. and Kim, Y.-J. (2018) Spinning Dust Emission from Circumstellar Disks and Its Role in Excess Microwave Emission. The Astrophysical Journal, 862, 116-131. https://doi.org/10.3847/1538-4357/aaccf0

[21] De Oliveira-Costa, A., Tegmark, M., Gutiérrez, C.M., Jones, A.W., Davies, R.D., Lasenby, A.N., Rebolo, R. and Watson, R.A. (1999) Cross-Correlation of Tenerife Data with Galactic Templates-Evidence for Spinning Dust? The Astrophysical Journal, 527, L9-L12. https://doi.org/10.1086/312384

[22] Finkbeiner, D.P., Schlegel, D.J., Frank, C. and Heiles, C. (2002) Tentative Detection of Electric Dipole Emission from Rapidly Rotating Dust Grains. The Astrophysical Journal, 566, 898-904. https://doi.org/10.1086/338225

[23] Watson, A., Rebolo, R., Rubĩno-Martín, J.A., Hildebrandt, S., Gutiérrez, C.M., Fernández-Cerezo, S., Hoyland, R.J. and Battistelli, E.S. (2005) Detection of Anomalous Microwave Emission in the Perseus Molecular Cloud with the Cosmosomas Experiment. The Astrophysical Journal, 624, L89-L92. https://doi.org/10.1086/430519

[24] Todorovíc, M., Davies, R.D., Dickinson, C., Davis, R.J. and Cleary, K.A. (2010) A 33 GHz VSA Survey of the Galactic Plane from 27 to 46 Degrees. Monthly Notices of the Royal Astronomical Society, 406, 1629-1643. https://doi.org/10.1111/j.1365-2966.2010.16809.x

[25] Scaife, A., Green, D.A., Battye, R.A., Davies, R.D., Davis, R.J., Dickinson, C., Franzen, T., Génova-Santos, R., Grainge, K., Hafez, Y.A., Hobson, M.P., Lasenby, A., Pooley, G.G., Rajguru, N., Rebolo, R., Rubiño-Martin, J.A., Saunders, R.D.E., Scott, P.F., Titterington, D., Waldram, E. and Watson, R.A. (2007) Constraints on Spinning Dust towards Galactic Targets with the Very Small Array: A Tentative Detection of Excess Microwave Emission towards 3C396. Monthly Notices of the Royal Astronomical Society, 377, L69-L73. https://doi.org/10.1111/j.1745-3933.2007.00305.x

[26] Casassus, S., Dickinson, C., Cleary, K., Paladini, R., Etxaluze, M., Lim, T., White, G.J., Burton, M., Indermuehle, B., Otmar Stahl, O. and Roche, P. (2006) Centimeter-Wave Continuum Radiation from the Ophiuchi Molecular Cloud. Monthly Notices of the Royal Astronomical Society, 391, 1075-1090. https://doi.org/10.1111/j.1365-2966.2008.13954.x

[27] Dickinson, C., Davies, R.D., Allison, J.R., Bond, J.R., Casassus, S., Cleary, K., Davis, R.J., Jones, M.E., Mason, B.S., Myers, S.T., Pearson, T.J., Readhead, A.C.S., Sievers, J.L., Taylor, A.C., Todorovíc, M., White, G.J. and Wilkinson, P.N. (2009) Anomalous Microwave Emission from the H II Region RCW175C. The Astrophysical Journal, 690, 1585-1589. https://doi.org/10.1088/0004-637X/690/2/1585

[28] Davies, R.D., Dickinson, C., Banday, A.J., Jaffe, T.R., Górski, K.M. and Davis, R.J. (2006) A Determination of the Spectra of galactic Components Observed by the Wilkinson Microwave Anisotropy Probe. Monthly Notices of the Royal Astronomical Society, 370, 1125-1139. https://doi.org/10.1111/j.1365-2966.2006.10572.x

[29] Ĺopez-Caraballo, C.H., Rubĩno-Martín, J.A., Rebolo, R. and Génova-Santos, R. (2011) Constraints on the Polarization of the Anomalous Microwave Emission in the Perseus Molecular Complex from Seven Year WMAP Data. The Astrophysical Journal, 729, 25-35. https://doi.org/10.1088/0004-637X/729/1/25 
[30] Génova-Santos, R., Rebolo, R., Rubiño-Martin, J.A., Ĺopez-Caraballo, C.H. and Hildebrandt, S.R. (2011) Detection of Anomalous Microwave Emission in the Pleiades Reflection Nebula with WMAP and the COSMOSOMAS Experiment. The Astrophysical Journal, 743, 67-78. https://doi.org/10.1088/0004-637X/743/1/67

[31] Stevenson, M.A. (2014) Derivation of Analytical Approximation of the Spectrum of Spinning Dust Emission. The Astrophysical Journal, 781, 113-124. https://doi.org/10.1088/0004-637X/781/2/113

[32] Draine, B.T. and Hensley, B.S. (2016) Quantum Suppression of Alignment in U1trasmall Grains: Microwave Emission from Spinning Dust Will Be Negligibly Polarized. The Astrophysical Journal, 831, 59-73.

https://doi.org/10.3847/0004-637X/831/1/59

[33] Hoang, T. and Tram, L.N. (2019) Dust Rotational Dynamics in C-Shocks: Rotational Disruption of Nanoparticles by Stochastic Mechanical Torques and Spinning Dust Emission. The Astrophysical Journal, 877, 36-52.

https://doi.org/10.3847/1538-4357/ab1845

[34] Ali-Haïmoud, Y. (2013) Spinning Dust Radiation: A Review of the Theory. Advances in Astronomy, 2013, Article ID: 462697. https://doi.org/10.1155/2013/462697

[35] Bertram, E., Glover, S.C.O., Clark, P.C., Ragan, S.E. and Klessen, R.S. (2016) Synthetic Observations of Molecular Clouds in a Galactic Center Environment: I. Studying Maps of Column Density and Integrated Intensity. Monthly Notices of the Royal Astronomical Society, 455, 3763-3780. https://doi.org/10.1093/mnras/stv2619 